\title{
Resultados del soporte nutricional preoperatorio en pacientes con neoplasia gastrointestinal en el Hospital General de Medellín
}

\author{
Results of preoperative nutritional support in patients with \\ gastrointestinal neoplasia at Medellín's General Hospital
}

\author{
Sebastián Grisales Ramírez ', Marta Ofelia Correa Posada ', Carolina Tobón Echavarría ${ }^{1,2}$, \\ Andrés Felipe Acevedo Betancur ${ }^{3}$, Marta Elena Muñoz Peláez ${ }^{4}$, Andrea Castrillón Castrillón ${ }^{1}$
}

Recibido: 29 de diciembre 2017. Aceptado para publicación: 1 de marzo 2018 https://doi.org/10.35454/rncm.v1n1.072

\section{Resumen}

Introducción: la malnutrición severa es un factor de riesgo para desenlaces adversos incluyendo mayor mortalidad en los pacientes con neoplasia gastrointestinal que son llevados a cirugía. Una intervención oportuna con nutrición preoperatoria disminuye la morbilidad y mortalidad en este grupo de pacientes.

Objetivo: realizar una caracterización clínica de pacientes sometidos a un programa de evaluación y recuperación nutricional sometidos a cirugía oncológica con intención curativa o paliativa, en el hospital General de Medellín y determinar los resultados en los pacientes intervenidos nutricionalmente en cuanto a morbilidad postoperatoria.

Metodología: estudio descriptivo retrospectivo de corte transversal, entre enero de 2014 y enero de 2016. Las variables analizadas fueron el estado nutricional, tipo de cirugía, tipos de histología y el tiempo de estancia.

Resultados: se incluyeron 48 pacientes en el estudio. La mayoría recibió soporte nutricional por vía enteral. El $80 \%$ de la mortalidad ocurrió en pacientes con desnutrición severa. El grado de desnutrición se correlacionó con las complicaciones postoperatorias, principalmente infección, mayor estancia hospitalaria, reingreso y requerimiento de $\mathrm{UCl}$ y ventilación mecánica.

Conclusiones: el soporte nutricional preoperatorio en pacientes oncológicos con neoplasia gastrointestinal puede ser una medida que ayude a la disminución de la morbilidad postoperatoria.

Palabras clave: malnutrición, estado nutricional, soporte nutricional, terapia nutricional, neoplasias gastrointestinales.

1 Facultad de Medicina, Universidad CES, Medellín, Colombia.

2 Universidad de Antioquia, Medellín, Colombia.

\section{Summary}

Introduction: Severe malnutrition is a risk factor for adverse outcomes, including an increased mortality in patients with gastrointestinal tract neoplasia who are taken to surgery. Timely intervention involving preoperative nutrition therapy improves morbidity and mortality in this group of patients.

Objective: To perform a clinical characterization of patients undergoing nutritional evaluation and recovery program undergoing oncological surgery with curative or palliative intention at Medellín's General Hospital, and determine the results in patients who received nutritional intervention in terms of postoperative morbidity.

Methodology: Descriptive retrospective cross-sectional study between January, 2014 and January, 2016. The analyzed variables were nutritional status, kind of surgery, types of histology and length of hospital stay.

Results: 48 patients were included in this study. Most of the patients received enteral nutritional support. $80 \%$ of the mortality occurred in several malnourished patients. The degree of malnutrition correlated to postoperative complications, mainly infection, greater length of hospital stay, readmission, and greater intensive care and mechanical ventilation requirements.

Conclusions: Preoperative nutritional support in the patient with gastrointestinal neoplasia can be an intervention to improve postoperative morbidity.

Keywords: Malnutrition; Nutritional status; Nutritional support; Nutritional therapy; Gastrointestinal neoplasia.

\footnotetext{
3 Servicio de Cirugía General, Hospital General de Medellín, Medellín, Colombia.

4 Servicio de Nutrición, Hospital General de Medellín, Medellín, Colombia.
} 


\section{INTRODUCCIÓN}

La malnutrición es una enfermedad prevalente en el mundo, especialmente en países en vía de desarrollo. El espectro de la misma comprende desde el déficit hasta el exceso de nutrientes, este último en el caso de los pacientes con obesidad que pueden tener sarcopenia ${ }^{(1)}$. Se ha establecido que la prevalencia de la malnutrición intrahospitalaria es de $50 \%$ en Estados Unidos ${ }^{(2)}, 21$ $\%$ en los países europeos ${ }^{(3)}$ y $27 \%$ en Gran Bretaña, este último en población quirúrgica ${ }^{(4)}$. En el caso de Latinoamérica, se encontró en Brasil una prevalencia de la desnutrición moderada y severa de $35 \%$ y $12 \%$ respectivamente en un estudio de 4.000 pacientes ${ }^{(5)}$. En Colombia en un estudio en el Hospital San Ignacio en Bogotá, $51 \%$ de los pacientes tenían desnutrición moderada y $20 \%$ severa ${ }^{(6)}$. Otras publicaciones en este país expusieron una prevalencia de la desnutrición hospitalaria de $63 \%{ }^{(7)}$ y $69,3 \%{ }^{(8)}$.

La inanición, la inflamación aguda y crónica son factores que pueden solaparse en el tiempo y se han asociado a la malnutrición intrahospitalaria ${ }^{(9)}$. En el subgrupo del paciente oncológico, la historia natural de la enfermedad progresa de manera vertiginosa, a lo que se suma el estado catabólico, caquexia e hiporexia, esta última dada por las caquexinas. Estos son considerados factores agravantes que llevan a las deficiencias nutricionales lo que hace que el acto quirúrgico tenga mayor potencial de morbilidad.

Se ha establecido que la malnutrición puede llegar a triplicar la mortalidad, la estancia hospitalaria ${ }^{(10)}$ y los desenlaces postoperatorios como infecciones, fístulas y filtraciones de las anastomosis, entre otras ${ }^{(5)}$, por tal motivo la intervención nutricional preoperatoria podría mejorar dichos resultados.

Algunos beneficios específicos que han sido atribuidos a la nutrición previa incluyen la reducción de las infecciones, mejor cicatrización, disminución en las infecciones del sitio operatorio (ISO) y filtración de la anastomosis, esta última en parte explicada por la disminución del estrés quirúrgico e inflamatorio y la prevención de la atrofia de las vellosidades intestinales ${ }^{(11-14)}$.

La Asociación Americana de Nutrición Parenteral y Enteral (ASPEN) ${ }^{(15)}$ y la Asociación Europea de Nutrición Clínica y Metabolismo (ESPEN) presentan guías en las cuales se propone la detección preoperatoria de la malnutrición y la intervención pertinente. Existen en el mercado varias fórmulas nutricionales para soporte, estas pueden ser desde suplementos poliméricos, oligoméricos, fórmulas especializadas y modulares ${ }^{(16)}$.
El objetivo principal de este estudio fue determinar las características nutricionales de los pacientes sometidos a un programa de evaluación y recuperación nutricional llevados a cirugía oncológica con intención curativa o paliativa, en el hospital General de Medellín. Otro objetivo fue evaluar los resultados en los pacientes intervenidos nutricionalmente en cuanto a morbilidad postoperatoria.

\section{METODOLOGÍA}

Se realizó un estudio descriptivo retrospectivo de corte transversal, donde se analizaron todos los pacientes hospitalizados en el Hospital General de Medellín, mayores de 18 años, de ambos sexos, entre enero de 2014 y enero de 2016 con diagnóstico de enfermedad maligna del tracto gastrointestinal. Se incluyeron aquellos pacientes que después de la estadificación requirieron cirugía curativa o paliativa. A todos se les realizó evaluación nutricional objetiva con parámetros bioquímicos y antropométricos cada 7 días.

Se clasificaron los sujetos si cumplían los criterios así: desnutrición leve: A. Albúmina $>2,9-3,4 \mathrm{~g} / \mathrm{dL}$, B. Peso $80 \%-89 \%$ del peso ideal y C. Peso $85 \%-95 \%$ del usual. Desnutrición moderada: A. Albúmina 2,5 - 2,9g/ dL, B. Peso entre $70 \%$ y $79 \%$ del peso ideal y C. Peso entre $75 \%$ y $84 \%$ del usual. Desnutrición severa: A. El paciente se encuentra $20 \%$ por debajo del peso usual o pérdida significativa de peso, B. Albúmina $<2,9 \mathrm{~g} / \mathrm{dL}$ y C. Signos de gasto muscular, o sobrepeso si tenían un índice de masa corporal elevado (IMC $>30 \mathrm{~kg} / \mathrm{m}^{2}$ ). Todos los pacientes del estudio recibieron soporte nutricional preoperatorio. El seguimiento se hizo hasta 30 días después del alta. Se analizaron las variables: el estado nutricional, tipo de cirugía, tipos de histología, el tiempo de estancia y las complicaciones postoperatorias. Se reportó el número de complicaciones postoperatorias en cada una de las categorías.

Debido al tamaño de la muestra no fue posible realizar evaluaciones estadísticas de asociación.

El estudio fue aprobado por el Comité de Ética del Hospital. El estudio adhirió a la resolución 8430 de 1993 del gobierno colombiano para la realización de trabajos de investigación en humanos.

\section{RESULTADOS}

Un total de 71 pacientes fueron analizados, 22 se excluyeron porque no eran susceptibles de manejo quirúrgico y uno removido porque no cumplió el número mínimo de días de terapia nutricional. Fueron incluidos 48 pacientes, $52,1 \%(\mathrm{n}=25)$ hombres y $47,9 \%(\mathrm{n}=23)$ mujeres. 
El promedio de edad fue 66 años (rango: 33 - 84). De la totalidad de pacientes, 28 presentaban comorbilidades y/o antedecente de tabaquismo o alcoholismo. Las características de la población en cuanto a comorbilidades y tipo de cirugía se pueden ver en la tabla 1.

El requerimiento administrado fue de $25-30 \mathrm{Kcal} / \mathrm{kg}$ y proteínas $1,5-2,0 \mathrm{~g} / \mathrm{kg}$. La vía de administración podía ser por vía oral, sonda avanzada NE (con fórmula Perative Abbott ${ }^{\oplus}$ ) o parenteral (NP) esta última a los pacientes que no podían tolerar vía oral o la nutrición avanzada. Los objetivos para llevar al paciente a cirugía: tener mínimo 7 días de terapia nutricional contando como día cero aquel que lograba alcanzar 100 $\%$ de la meta calórica, prealbúmina con tendencia al aumento, balance nitrogenado (BN) positivo o anabólico (balance nitrogenado $=$ nitrógeno administrado $(\mathrm{g} / 24 \mathrm{~h})$ - Nitrógeno eliminado $(\mathrm{g} / 24 \mathrm{~h})+4$. Se considera neutro $=0$, anabólico $\geq$ a 1 y catabólico $\leq-1$ ). Pacientes con desnutrición severa recibieron aporte calórico lo más cercano a 14 días, en caso de no cumplir lo anteriormente mencionado, eran llevados a cirugía después del día número 16 .

Las neoplasias más frecuentemente intervenidas fueron el adenocarcinoma gástrico $41,7 \%(\mathrm{n}=20)$, adenocarcinoma de colon $22,9 \%(\mathrm{n}=11)$, carcinoma de la

Tabla 1. Características clínicas de la población con tumores gastrointestinales e intervención quirúrgica en el HGM, 2015

\begin{tabular}{|l|r|c|}
\hline & n & $\%$ \\
\hline Comorbilidades y factores de riesgo & 28 & 58,3 \\
\hline Tabaco & 19 & 39,6 \\
\hline Hipertensión arterial & 14 & 29,2 \\
\hline $\begin{array}{l}\text { Enfermedad Pulmonar Obstructiva } \\
\text { Crónica }\end{array}$ & 8 & 16,7 \\
\hline Alcohol & 5 & 10,4 \\
\hline Diabetes Mellitus & 5 & 10,4 \\
\hline Hipotiroidismo & 2 & 4,2 \\
\hline Enfermedad coronaria & 2 & 4,2 \\
\hline Enfermedad renal crónica & 2 & 4,2 \\
\hline Tipo de Cirugía & 48 & 100 \\
\hline Esofagectomías & 4 & 8,3 \\
\hline Gastrectomías totales & 9 & 18,8 \\
\hline Gastrectomías subtotales & 13 & 27,1 \\
\hline Gastroyeyunostomía & 1 & 2,1 \\
\hline Pancreatoduodenectomías & 8 & 16,7 \\
\hline Hemicolectomías derecha & 8 & 16,7 \\
\hline Hemicolectomías izquierda & 1 & 2,1 \\
\hline Sigmoidectomías & 2 & 4,2 \\
\hline Colostomías derivativas & 1 & 2,1 \\
\hline Resección abdominoperineal & 1 & 2,1 \\
\hline
\end{tabular}

ampolla de váter 14,6 \% $(\mathrm{n}=7)$, carcinoma de esófago $10,4 \%(\mathrm{n}=5)$, leiomiosarcoma gástrico $2,1 \%(\mathrm{n}=1)$, carcinoma de recto $4,2 \%(\mathrm{n}=2)$ y carcinoma de la cabeza del páncreas $2,1 \%(n=1)$. De todos los anteriores, el $95,8 \%(\mathrm{n}=46)$ fueron resecables en el intraoperatorio y solo $4,2 \%(n=2)$ de los casos requirieron cirugía paliativa.

El estado nutricional preoperatorio de la población estudiada se encontró: adecuado en cinco pacientes $(10,4 \%)$, desnutrición leve en seis pacientes (12,5\%), moderada en cuatro pacientes $(8,3 \%)$, severa en 30 pacientes $(62,5 \%)$ y tres pacientes con sobrepeso $(6,3 \%)$.

La vía de administración de la nutrición fue la enteral en $97,9 \%(n=47), 58,3 \%(n=28)$ suplementación oral y $39,6 \%(n=19)$ sonda avanzada.

El tiempo de la administración de la nutrición fue 7 días, 33,3\% $(\mathrm{n}=16), 8$ - 15 días, 47,9\% $(\mathrm{n}=23)$ y más de 16 días, $18,8 \%(\mathrm{n}=9)$, de todos los anteriores $8,2 \%$ $(\mathrm{n}=4)$ no lograron ingerir $>80 \%$ de la meta calórica diaria en las primeras 72 horas por intolerancia inicial, secundario a una obstrucción al tracto de salida gástrico por adenocarcinoma en todos los casos previamente mencionados, la cual fue alcanzada posteriormente mediante alimentación por sonda nasoyeyunal avanzada.

En cuanto al BN, se logró anabolismo en 35,4\% ( $\mathrm{n}=$ 17) de los pacientes, neutro en $18,8 \%(\mathrm{n}=9)$ y catabolismo leve en $8,3 \%(n=4)$, no se evidenció catabolismo moderado ni severo. No se realizó BN en 37,5 \% $(\mathrm{n}=18)$ debido a que muchos de ellos recibieron nutrición oral lo cual dificultaba este cálculo.

El 14,6\% de los pacientes estuvieron menos de 19 días hospitalizados, $50 \%$ estuvo entre 20 y 39 días y el $35,4 \%$ más de 40 días. La relación entre el estado nutricional y la estancia hospitalaria, en Unidad de cuidados intensivos (UCI), Unidad de cuidados especiales (UCE) y días asociados a ventilación mecánica se pueden evidenciar en la tabla 2.

Las complicaciones postoperatorias más frecuentes y su relación con el tipo de desnutrición pueden ser vistas en la tabla 3. Las más comunes fueron las infecciones del sitio operatorio (ISO) de las cuales fueron superficiales $8,3 \%(n=4)$ y órgano espacio $20,8 \%(n=10)$, no se presentaron infecciones profundas ni infecciones cuando el paciente se encontraba adecuadamente nutrido, mientras que el mayor número de infecciones se presentaron en pacientes con desnutrición severa $(\mathrm{n}=8)$ seguido de desnutrición moderada $(\mathrm{n}=2)$ y de leve $(\mathrm{n}=3)$.

La mortalidad general del estudio fue de cuatro pacientes $(8,3 \%)$, tres de ellos con desnutrición severa, dos estuvieron relacionadas con pancreatoduodenec- 
Tabla 2. Relación del estado nutricional inicial y la estadía en hospitalización, unidad de cuidados intensivos (UCI), unidad de cuidados especiales (UCE) y los días de ventilación mecánica en los pacientes intervenidos por cáncer gástrico que recibieron intervención nutricional en el HGM según el estado de desnutrición (DNT)

\begin{tabular}{|c|c|c|c|c|c|c|}
\hline & $\begin{array}{c}\text { Normal } \\
\text { Media y } \\
\text { (Rango) }\end{array}$ & $\begin{array}{c}\text { DNT leve } \\
\text { Media y } \\
\text { (Rango) }\end{array}$ & $\begin{array}{c}\text { DNT moderada } \\
\text { Media y } \\
\text { (Rango) }\end{array}$ & $\begin{array}{c}\text { DNT severa } \\
\text { Media y } \\
\text { (Rango) }\end{array}$ & $\begin{array}{c}\text { Sobrepeso } \\
\text { Media y } \\
\text { (Rango) }\end{array}$ & $\begin{array}{c}\text { Total } \\
\text { Media y } \\
\text { (Rango) }\end{array}$ \\
\hline $\begin{array}{c}\text { Estancia } \\
\text { Hospitalaria }\end{array}$ & $29(16-49)$ & $54(19-112)$ & $53(33-73)$ & $32(11-75)$ & $30(20-50)$ & $36(11-112)$ \\
\hline Estancia UCI & $6(0-19)$ & $7,17(0-19)$ & $4,75(0-11)$ & $4,03(0-28)$ & $11(1-28)$ & $5,13(0-28)$ \\
\hline Estancia UCE & $2,4(1-4)$ & $3,5(0-10)$ & $5,25(0-18)$ & $2,13(0-9)$ & $2,33(0-7)$ & $2,6(0-18)$ \\
\hline $\begin{array}{c}\text { Días de ventilación } \\
\text { mecánica }\end{array}$ & $2,6(0-7)$ & $0,33(0-2)$ & $2,75(0-7)$ & $1,13(0-18)$ & $6,33(0-19)$ & $1,65(0-19)$ \\
\hline
\end{tabular}

Tabla 3. Número de complicaciones postoperatorias según el estado nutricional inicial en los pacientes intervenidos por cáncer gástrico que recibieron intervención nutricional en el HGM

\begin{tabular}{|l|c|c|c|c|c|}
\hline & $\begin{array}{c}\text { Normal } \\
\text { (n) }\end{array}$ & $\begin{array}{c}\text { DNT } \\
\text { Leve }\end{array}$ & $\begin{array}{c}\text { DNT } \\
\text { Moderada }\end{array}$ & $\begin{array}{c}\text { DNT } \\
\text { Severa }\end{array}$ & Sobrepeso \\
\hline ISO & 0 & 3 & 2 & 8 & 1 \\
\hline Reingreso & 1 & 0 & 0 & 3 & 1 \\
\hline Fístula & 0 & 3 & 2 & 3 & 1 \\
\hline Sepsis & 2 & 2 & 2 & 2 & 1 \\
\hline Delirium & 2 & 1 & 0 & 2 & 0 \\
\hline SDRA & 2 & 0 & 1 & 3 & 0 \\
\hline Arritmias & 0 & 1 & 0 & 2 & 0 \\
\hline $\begin{array}{l}\text { Disfunción } \\
\text { cardiaca }\end{array}$ & 1 & 0 & 1 & 1 & 0 \\
\hline $\begin{array}{l}\text { Disfunción } \\
\text { hepática }\end{array}$ & 1 & 0 & 0 & 1 & 1 \\
\hline $\begin{array}{l}\text { Lesión renal } \\
\text { aguda }\end{array}$ & 2 & 0 & 0 & 6 & 0 \\
\hline Ileus & 0 & 0 & 0 & 3 & 1 \\
\hline Coagulopatía & 0 & 0 & 0 & 1 & 1 \\
\hline Mortalidad & 1 & 0 & 0 & 3 & 0 \\
\hline
\end{tabular}

tomía, uno con esofagectomía y uno con gastrectomía total. Luego del alta, 12,5 \% de los pacientes reingresó a los 30 días.

\section{DISCUSIÓN}

La malnutrición hospitalaria, en particular en el paciente con cáncer, es una enfermedad altamente prevalente. En este estudio la prevalencia fue del 62,5\%, en concordancia con lo encontrado en estudios previos en Estados Unidos y en Bogotá( ${ }^{(1,6)}$, donde se evidencia malnutrición severa de $50 \%$ y $51 \%$ respectivamente.
Estudios previos muestran que la mortalidad en estos pacientes puede aumentarse en forma sustancial. En un análisis de regresión multivariado publicado por I. Correia, et al. ${ }^{(10)}$, se reconoce que la mortalidad puede ser tres veces mayor en malnutridos $(12,4 \%$ frente a $4,7 \%$ ) lo cual coincide con los hallazgos de este estudio, donde la mortalidad fue de $10 \%$ de pacientes con malnutrición severa. Es importante resaltar que solo un paciente falleció con adecuado estado nutricional y 3 de 5 tenían más de 74 años.

En el mismo estudio ${ }^{(10)}$ la estancia hospitalaria en pacientes malnutridos en relación con los pacientes eutróficos fue $16,7 \pm 24$ vs 10,1 \pm 11,7 días, esto llevaría a un aumento del costo en atención en 308,9 \%. En el grupo de pacientes del presente estudio la mayor diferencia en la estancia hospitalaria fue dada entre pacientes eutróficos en relación con los leve y moderadamente malnutridos (media de 29 vs 54 y 53 respectivamente). Dicha magnitud podría ser en parte explicada por el tiempo necesario para la administración de la nutrición y llegar a la meta definida, así como también por el tiempo de espera de la autorización administrativa de las cirugías por parte de las Entidades Prestadoras de Salud (EPS), además de lo antes mencionado, la estancia en UCI de los pacientes con sobrepeso fue de casi el doble al relacionarla con los pacientes eutróficos (media de 11 vs 6 días) explicado principalmente por la ventilación mecánica. En adición, la media general de estancia en UCI fue de 5 días $(0$ - 28) y en UCE de 2,6 días (0 - 18) y muchos de los pacientes eran trasladados de UCI a sala de hospitalización general. La probable explicación de este contrasentido es la dificultad para tener disponible la cama en UCE ya que al 
ser un hospital de tercer nivel y centro de referencia en el departamento de Antioquia se presenta una gran ocupación de sus servicios.

La terapia nutricional ha demostrado ser eficaz. En un estudio multicéntrico de 1007 pacientes quirúrgicos generales en Estados Unidos, se demostró que esta reduce el tiempo de hospitalización a 11,9 días (vs 14,8), lo cual reduce el costo de la atención médica de 38,578 USD a 34,602 USD ${ }^{(17)}$. Los dos resultados alcanzaron valor estadístico $(\mathrm{p}<0,05)$. Esta reducción de costos sería de gran beneficio para el sistema de salud colombiano.

Un hallazgo importante en nuestro estudio fue que se evidenció un incremento de 2,4 veces de la media de días en ventilación mecánica en los pacientes con sobrepeso/obesidad en comparación con los pacientes eutróficos, esto es explicado por el gran panículo adiposo que produce un aumento de la presión intra abdominal, lo que lleva a la disminución de la capacitancia pulmonar presentando como consecuencia el aumento de la presión pico y meseta en la ventilación mecánica dificultando su proceso de extubación y prolongando los días de ventilación.

$\mathrm{Al}$ relacionar pacientes eutróficos con pacientes desnutridos solo se pudo encontrar un aumento de los días máximos de ventilación mecánica pero fue muy similar a la media.

Los estudios relacionados con la morbilidad indican que esta es mayor en pacientes malnutridos, ya que por ejemplo, puede haber una incidencia de $27 \%$ vs $16 \%$ al compararlo con los pacientes eutróficos (RR 1,60; IC 1,2 a 2,14; $\mathrm{p}<0,05)$ como lo muestra el estudio brasileño de Waitzberg, et al. ${ }^{(5)}$.

La morbilidad general de este estudio fue de $45 \%$, y la principal complicación la infección del sitio operatorio. Si se excluye dicha complicación, la morbilidad total se reduce a $16 \%$. Una posible explicación para esto sería que la mayoría de infecciones se presentaron en pacientes severamente desnutridos lo cual los lleva a un compromiso de su sistema inmune.

En 18,8\% de los pacientes se presentó filtración de la anastomosis expresada como fístula y no se presentaron filtraciones libres a cavidad abdominal. En un estudio retrospectivo de 114 pacientes con enfermedad de Crohn se compararon pacientes con terapia nutricional preoperatoria $v s \sin$ terapia preoperatoria, se evidenció que el número de filtraciones de la anastomosis se redujo de $17,9 \%$ a 2,3 \% p 0,023 y menor número de ostomías derivativas $(22,8 \% \text { vs } 40,9 \%)^{(18)}$. Cabe anotar que el estado catabólico y la inanición de los pacientes onco- lógicos lleva a un estado de malnutrición mayor que en estos pacientes.

La continuación de la nutrición postoperatoria es clave en la recuperación del paciente. En los últimos años bajo las directrices de los protocolos ERAS (por sus siglas en inglés Enhanced Recovery After Surgery), se ha implementado la nutrición postoperatoria temprana y esto ha llevado a disminuir el número de complicaciones generales ${ }^{(19)}$. En este estudio todos los pacientes continuaron con aporte calórico adecuado el cual se inició en las $24-48 \mathrm{~h}$ posteriores al acto quirúrgico.

Se debe tener en cuenta que la nutrición preoperatoria es una preparación para que un paciente pueda ser sometido a un acto quirúrgico y un estrés postoperatorio, más que una verdadera corrección de su estado nutricional basal el cual tomaría meses en ser corregidos.

Hubo varias limitaciones en este estudio, primero es el tamaño de la muestra, ya que al ser un estudio que involucra varios procedimientos quirúrgicos requeriría una muestra de pacientes mucho mayor. Esto impidió hacer posible la realización de evaluaciones estadísticas de asociación.

Segundo, al ser un estudio retrospectivo dependiente de la historia clínica se pudo presentar un sesgo de información por una posible omisión de datos importantes. Para controlar este sesgo se intentó tener un formato estándar para la recopilación de los datos y así disminuir este riesgo. Tercero, la evaluación del $\mathrm{BN}$ no fue posible en todos los pacientes ya que es necesario conocer con exactitud el aporte de nitrógeno en relación con la excreción del mismo en orina lo cual es difícil de calcular con nutrición oral.

\section{CONCLUSIONES}

La malnutrición es una enfermedad con alta prevalencia, en especial en pacientes oncológicos intervenidos quirúrgicamente lo cual fue demostrado en la población del estudio. La vía de nutrición enteral sigue siendo la forma más frecuente de administración de la nutrición. Los pacientes desnutridos y en sobrepeso presentan mayor requerimiento de ventilación mecánica y aumento de los tiempos de estadía hospitalaria en UCI.

Se requieren estudios prospectivos y con una mayor muestra para establecer asociaciones y poder evaluar el efecto del soporte nutricional en estos pacientes.

\section{Conflicto de intereses}

Ninguno 


\section{Financiación}

Los autores principales asumieron la totalidad de la financiación del estudio.

\section{Referencias bibliográfícas}

1. Allison SP. Malnutrition, disease, and outcome. Nutrition. 2000;16:590-3.

2. Gamble Coats K, Morgan S, Bartolucci A, Weinsier R. Hospital-associated malnutrition: A reevaluation 12 years later. J Am Diet Assoc. 1993;93:27-33.

3. Kondrup J, Sorensen J. The Magnitude of the Problem of Malnutrition in Europe. In: Elia M, Bistrian B (eds). The Economic, Medical/Scientific and Regulatory Aspects of Clinical Nutrition Practice: What Impacts What? Basel: Nestec. 2009; 12:1-14.

4. McWhirter J, Pennington C. Incidence and recognition of malnutrition in hospital. BMJ. 1994;308:945-8.

5. Waitzberg D, Caiaffa W, Correia M. Hospital malnutrition: the Brazilian national survey (IBRANUTRI): a study of 4000 patients. Nutrition. 2001;17:573-80.

6. Manosalva D. Desnutrición en Hospital San Ignacio en Bogotá. Universidad Javeriana. 1990. Tesis de Grado. Disponible en: http://www.citethisforme.com/topic-ideas/ biology/nutricion-52867717

7. Giraldo Giraldo NA, Múnera García NE, Espitaleta Marrugo V, Pinerez LM. Prevalence of malnutrition and evaluation of dietary treatment for adult hospitalized patients in a public institution of high complexity. Perspect Nut Hum. 2007;9:37e47.

8. Agudelo R, Giraldo NA, Aguilar NL, Restrepo B, Vanegas M, Alzate $\mathrm{S}$, et al. Incidence of nutritional support complications in patient hospitalized in wards. multicentric study. Colomb Med. 2012;43:147e53. Consultado el 2 de diciembre 2017.

9. Jensen G, Mirtallo J, Compher C, Dhaliwal R, Forbes A, Grijalba R, et al. Adult Starvation and Disease-Related Malnutrition. JPEN J Parenter Enteral Nutr. 2010;34:156-9.

10. Correia MI. The impact of malnutrition on morbidity, mortality, length of hospital stay and costs evaluated through a multivariate model analysis. Clin Nutr. 2003;22:235-9.

11. Ward N. Nutrition support to patients undergoing gastrointestinal surgery. Nutr J. 2003;2(1).

12. Bozzetti F. Peri-operative nutritional management. Proc. Nutr. Soc. 2011;70:305-10. 2011;70:305-10.

13. Braunschweig CL, Levy P, Sheean PM, Wang X. Enteral compared with parenteral nutrition: a meta-analysis. Am J Clin Nutr. 2001;74:534-42.

14. Beier-Holgersen R, Boesby S. Influence of postoperative enteral nutrition on postsurgical infections. Gut. 1996;39:833-5.

15. August D, Huhmann M. A.S.P.E.N. Clinical Guidelines: Nutrition Support Therapy During Adult Anticancer Treatment and in Hematopoietic Cell Transplantation. JPEN J Parenter Enteral Nutr. 2009;33(5):472-500.

16. Campbell S. An Anthology of Advances in Enteral Tube Feeding Formulations. Nutr Clin Pract. 2006;21:411-5.

17. Neumayer L, Smout R, Horn H, Horn S. Early and Sufficient Feeding Reduces Length of Stay and Charges in Surgical Patients. J Surg Res. 2001;95:73-7.

18. Guo Z, Guo D, Gong J, Zhu W, Zuo L, Sun J, et al. Preoperative Nutritional Therapy Reduces the Risk of Anastomotic Leakage in Patients with Crohn's Disease Requiring Resections. Gastroenterology Research and Practice. 2016:1-7.

19. Ljungqvist O, Scott M, Fearon K. Enhanced Recovery After Surgery. JAMA Surgery. 2017;152:92. 